\title{
Stage IIIB Uterine Corpus Endometrial Stromal Sarcoma AJCC v8
}

National Cancer Institute

\section{Source}

National Cancer Institute. Stage IIIB Uterine Corpus Endometrial Stromal Sarcoma A/CC v8. NCI Thesaurus. Code C139889.

Stage IIIB includes: T3b, N0, M0. T3b: Uterine corpus endometrial stroma sarcoma with tumor infiltrating abdominal tissues at more than one site. N0: No regional lymph node metastasis. M0: No distant metastasis. (AJCC 8th Ed.) 